DOI $10.35381 / \mathrm{cm} . v 5 i 9.143$

\title{
El Mercado virtual de productos de primera necesidad desde la perspectiva comercial
}

\section{The feasibility of promoting a virtual market of products of first need from the commercial perspective}

\author{
Alfredo Mauricio Astudillo Mamarandi \\ aastudillo@unach.edu.ec \\ Universidad Nacional de Chimborazo Riobamba \\ Ecuador \\ https://orcid.org/0000-0001-5204-4927 \\ Mayra Gissela Lucio Solano \\ mlucio@ucem.com.ec \\ Unión Cementera Nacional-Tesorería, Riobamba \\ Ecuador \\ https://orcid.org/0000-0002-7330-2769 \\ Santiago Barriga \\ sbarriga@unach.edu.ec \\ Universidad Nacional de Chimborazo Riobamba \\ Ecuador \\ https://orcid.org/0000-0001-5527-148X
}

Recibido: 18 de marzo del 2019

Aprobado: 01 de mayo del 2019

\section{RESUMEN}

El presente trabajo de investigación tiene el objetivo de plantear un análisis dentro del ámbito comercial, partiendo de un prototipo de tienda virtual que oferta productos agrícolas, de los productores minoristas asociados al MAGAP - Chimborazo, para lo cual se tomó 3 contextos importantes como: i) investigación de mercado, con la implementación de 271 encuestas para determinar la aceptación del usuario a nuevas 
líneas de comercialización y la aceptación del entorno web en base a un test de usabilidad, ii) el tamaño del mercado, basado en un estudio descriptivo cuantitativo, para determinar el piso y el techo de producción de las organizaciones participantes y iii) la competencia, en donde se utilizó instrumentos de evaluación descriptiva para determinar fortalezas y debilidades de la nueva línea de comercialización.

Descriptores: Producción agrícola; Prototipo; Tienda virtual.

\begin{abstract}
The present research work has the objective of presenting an analysis within the commercial field, starting from a prototype of a virtual store that offers agricultural products, from the retail producers associated to MAGAP - Chimborazo, for which 3 important contexts were taken as: i ) market research, with the implementation of 271 surveys to determine user acceptance of new marketing lines and acceptance of the web environment based on a usability test, ii) market size, based on a quantitative descriptive study, to determine the floor and the production ceiling of the participating organizations and iii) the competition, where descriptive evaluation instruments were used to determine strengths and weaknesses of the new commercialization line.
\end{abstract}

Descriptors: Agricultural production; Prototype; On-line shop.

\title{
INTRODUCCIÓN
}

La distribución de productos agrícolas en los mercados y hogares de países en de desarrollo, están muy marcada en cuanto a otros bienes derivados del sector industrial, una de las causas predominantes son los intermediarios, ellos hacen que los productos agrícolas tengan costos elevados escudándose en factores externos como origen, temporada, calidad e incluso presentación, un factor para este fenómeno son sus mercados primarios, generadores de materia prima no procesada industrialmente.

Tomando en cuenta los niveles administrativos del país, la zona 3 integrado por provincias de acuerdo a una contigüidad geográfica, cultural y económica (Cotopaxi, Tungurahua, Chimborazo y Pastaza) es considerada como un centro de acopio agrícola 
y enclave comercial, por su ubicación y características de producción agrícola, destacando entre sus principales actividades económicas la producción agropecuaria, comercial y la producción manufacturera (GAD Provincial de Chimborazo, 2015). Sin embargo, este mercado no es muy dinámicos sobre todo en el área agrícola, por el acopio excesivo de intermediarios, los cuales se llevan la mayor ganancia por comercializar dichos productos dentro y fuera de la ciudad.

En Chimborazo y sobretodo en la ciudad de Riobamba, productores agrícolas rurales asesorados y auspiciados por el Ministerio de Agricultura y Ganaderías han puesto en marcha una especie de redes comerciales, proyectos que permita a los pequeños y medianos productores agrícolas vender sus productos directamente a consumidores finales, por medio de ferias en lugares considerados estratégicos dentro de la ciudad de Riobamba. Esto ha permitido una reducción de la intermediación, pero son puntos focales muy pequeños y de poco desarrollo. Bajo estos antecedentes, se busca crear un escenario ideal para el desarrollo agrícola, una feria constante, donde se pueda vender al por mayor y menor, pero al tener que ganar espacios dentro de la ciudad, basados en tramitologías burocráticas extensas, hace muy difícil llegar a más gente.

Por otro lado, la tecnología hace tendencia en todo ámbito y esto ha ocasionado que el ser humano cambie sus hábitos de consumo y convivencia dando paso a una economía digital, en donde mucho tiene que ver la intervención del gobierno, casos explícitos como Noruega, Suiza, Finlandia y Dinamarca quienes son catalogados como países mejor puntuados en el Informe sobre Evolución Digital en el Mundo 2017 del "Digital Planet 2017". También Estados Unidos (Chakravorti \& Shankar Chaturvedi, 2017), es pionero en invertir en la digitalización, en infraestructura web como en instituciones de desarrollo web. Esto se refleja en el incremento incontrolable de usuarios de internet; solo en Sur América hay más de 229 millones de usuarios de internet vinculados a redes sociales y representan el 39,3\% de la totalidad de usuarios en el continente (Camara, 2017). 


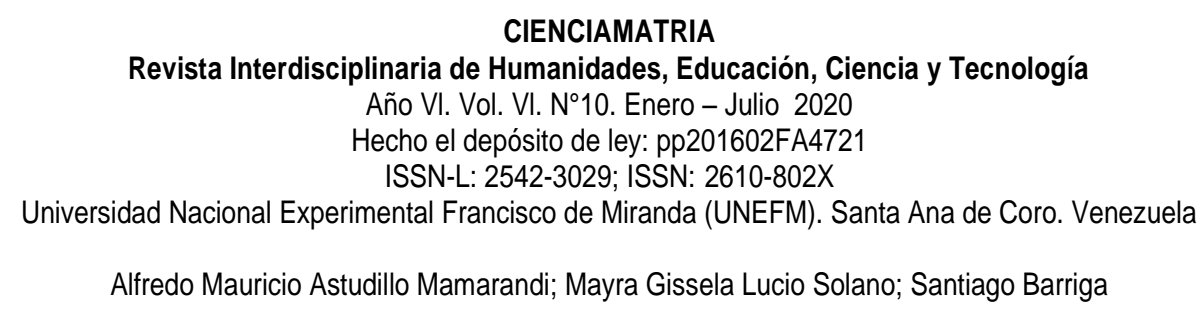

En el Ecuador, hasta el 2016, aproximadamente el 36\% de los hogares ecuatorianos cuentan con acceso al internet y de las cuales la cuarta parte de la población lo hace por medio inalámbrico (Smartphone, Tablet, portátiles), además si se toma como referencia el acceso al internet por áreas, lo urbano tiene mayor crecimiento con respecto a lo rural con un incremento de 3 puntos porcentuales desde el 2015 (INEC, 2016). La provincia de Chimborazo en uso del internet, se encuentra por debajo de la media de la población, sus factores pueden ser diversos como cambiantes, por cuestión de cobertura y acceso al servicio de internet. Al mismo tiempo refleja que existe un público cautivo muy importante a ser tomado en cuenta para futuros proyectos WEB de cualquier índole.

\section{DESARROLLO}

Con los antecedentes antes mencionados, por un lado, la necesidad de comercializar productos agrícolas por medio de un ente gubernamental y por otro el creciente uso del internet, hace una mezcla ideal para nuevos negocios dentro de la provincia. Y por qué no pensar como un ejercicio piloto para ejemplo de las demás zonas productoras de la región y del país. Por ese motivo esta investigación tiene el objetico de plantear un estudio preliminar para medir la factibilidad de impulsar un mercado virtual basado en un prototipo funcional de Tienda Virtual, en donde el intercambio transaccional sea los productos agrícolas.

Para eso hay que entender primero una situación muy importante dentro del entorno de estudio. Se trabajó con más de 150 agricultores, organizados en 2 grupos asociados de pequeña y mediana producción agrícola; $\mathrm{CORPOCOSACH}$ (integrada por 13 parroquias rurales de la ciudad de Riobamba) y AGROECOLÓGICA (integrada por 4 cantones de la provincia de Chimborazo), los productos de mayor comercialización son las hortalizas, legumbres, frutas, harinas, lácteos, huevos, miel y polen.

Desde el ámbito comercial es muy importante saber la apreciación del cliente, más aún cuando no está familiarizado con la comercialización en línea de productos de primera 


\section{CIENCIAMATRIA \\ Revista Interdisciplinaria de Humanidades, Educación, Ciencia y Tecnología \\ Año Vl. Vol. VI. №10. Enero - Julio 2020 \\ Hecho el depósito de ley: pp201602FA4721 \\ ISSN-L: 2542-3029; ISSN: 2610-802X \\ Universidad Nacional Experimental Francisco de Miranda (UNEFM). Santa Ana de Coro. Venezuela \\ Alfredo Mauricio Astudillo Mamarandi; Mayra Gissela Lucio Solano; Santiago Barriga}

necesidad, por eso fue necesario realizar estudios preliminares dentro de la concepción de la usabilidad para una plataforma web, con la ayuda de consultorías externas y el uso del análisis heurístico se pudo definir una propuesta firme de la plataforma WEB a ser estudiada. La evaluación heurística es muy utilizada en etapas tempranas en proyectos de desarrollo de entornos web, es común utilizar prototipos funcionales por lo económica frente a otras metodologías (Gonzáles, Pascual, \& Lorés, 2006). Una de sus mayores ventajas es: identificar los errores más comunes en el interfaz del usuario, ya que el experto analiza y señala dicha interfaz, cotejando con una guía de elementos básicos de diseño proporcionada por los creadores de la plataforma web (Catalán Vega, 2014), así mismo, Perozo Martín \& Chirinos Martínez (2019), agregan que esto permite ejecutar un modelo organizado de gestión, aunado a la experiencia de Colina (2018), quien destaca que la tecnología permite generar un negocio de mayor efectividad económica.

\section{METODOLOGÍA}

Una vez obtenido un prototipo funcional es necesario aplicar una evaluación comercial con los potenciales clientes, esto ayudará a detectar actitudes de los consumidores, problemas $u$ oportunidades dentro del mercado, entre otras. Los expertos de la mercadotecnia declaran que hay características que define una buena investigación de mercados, entre ellas, el uso de métodos científicos, la creatividad de la investigación y uso de métodos múltiples, entre los más importantes (Kotler \& Keller, 2012). En este punto se utiliza varias metodologías y técnicas de análisis de datos, por ser uno de los más importantes en la investigación. Es así que este estudio genera tres momentos de estudio, cada uno arrojara distintos resultados cuantitativos y cualitativos, pero en conjunto generaran lineamientos de decisión y comportamiento frente a un nuevo servicio, es así que se analiza lo siguiente:

a) Investigación de Mercado.

b) El tamaño del Mercado (demanda). 
c) La Competencia (Oferta).

a) Características generales de la investigación de mercado: Se utilizó la encuesta como parte de una investigación descriptiva, la misma que se ejecutó en la ciudad de Riobamba, en el mes de octubre del año 2018. El grupo de estudio escogido se determinó por factores macroeconómicos, Población Económicamente Activa (PEA). El fin de la investigación es comercializar productos agrícolas de necesidad masiva, en tal razón la muestra no refleja el tamaño del mercado.

\section{POBLACIÓN Y MUESTRA}

Según datos oficiales del INEC la Ciudad de Riobamba posee el $82 \%$ aproximado de la población de la provincia de Chimborazo, de las cuales aproximadamente unos 70.575 habitantes están dentro de la PEA. Al conocer la población objetivo, se abstrae una muestra de tipo probabilística aleatoria simple. Se elige este procedimiento de muestreo para garantizar que cada elemento de la población tenga la misma posibilidad de ser incluido (Jacquer, 2009). Como la población no supera los 100.000 por unidad de muestra, se aplica la siguiente fórmula:

$$
n=\frac{Z^{2} p q N}{N E^{2}+Z^{2} p q}
$$

Figura 1. Fórmula a aplicar para el tamaño de la muestra

Considerando la formula aplicada con una población de 70.575 habitantes (PEA), con un nivel de confianza del $90 \%$, con una variabilidad del 0.5 y un margen de error del $5 \%$, el segmento a ser estudiado es de 271 habitantes a ser encuestados en la ciudad de Riobamba.

\section{Esquema metodológico e instrumental de la investigación de mercado}


Con el fin de buscar mejorar las líneas de comercialización de las organizaciones que trabajan con el MAGAP Chimborazo, es necesario saber el grado de aceptación de la ciudadanía frente a un nuevo servicio en línea, para lo cual se planteó un método cuantitativo y como técnica la encuesta en 2 escenarios y se preparó instrumentos (cuestionario) para la recolección de datos por cada uno.

El primer escenario planteaba la posibilidad de aceptación de la página virtual el cual consta de 3 ítems de información general (Preguntas Cerradas) y 11 ítems de información específica (Escalas de Likert). Por medio de un piloto de pudo categorizar la prueba de confiabilidad del instrumento (Alfa de Cronbach $=0.837$ ), en este contexto se menciona que el rango aceptable debe ser mayor a 0.7 (Navarro, 2017). Considerando estos criterios se puede determinar que la consistencia interna de los ítems generales e individuales es buenos y están altamente correlacionados.

El segundo escenario plantea un Test de Usuario orientado a la exploración, contiene 23 ítems (Checklists) enfocados a diferentes ámbitos de: Identidad, Contenido, Navegación, Grafica Web, Feedback y Utilidad (Almazán \& Camus), guía general para la evaluación de la usabilidad de un sitio web (Montero \& Fernández, 2003). Con la aplicación de la encuesta se pretende identificar al usuario "tipo", y si el planteamiento de los desarrolladores y diseñadores en el aspecto del producto web son correctas (haciendo referencia a los resultados del estudio heurístico) desde la percepción del usuario (Catalán Vega, 2014). Además, fue necesario adaptar un modelo de test de usuario aplicado para la Biblioteca del Congreso de Chile, realizado por Felipe Almazán (Biblioteca del Congreso de Chile) y Juan. C. Camus (www.usando.info) enfocados a los ámbitos de:

- Identidad, las 5 preguntas fueron planteadas en base al reconocimiento del usuario frente a elementos identificadores relacionados a la empresa y su imagen corporativa y lo que buscan es la primera impresión del usuario cuando mira la pantalla inicial. 


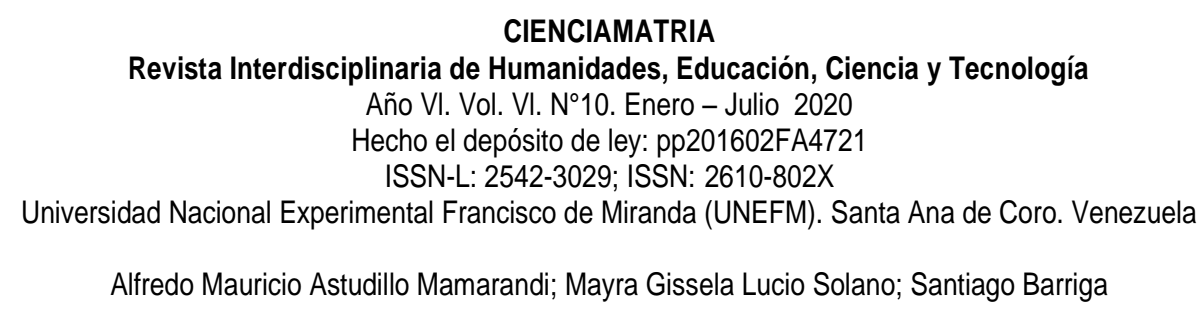

- Contenido, las 5 preguntas de la sección hace énfasis en las pantallas de inicio y de los infoproductos, se pretende verificar el funcionamiento del sitio, la organización de la información y si los prototipos ayudan a entender lo que muestra la página (que ofrece en sí).

- Navegación, 6 preguntas planteadas de una forma que: el usuario localice intuitivamente la información para navegar por el sitio, reconozca los íconos de enlaces como redes sociales, el carro de compra y pueda regresar a la página central 0 al de infoproductos.

- Grafica Web, las 3 preguntas planteadas en esta sección establece si la información gráfica ayuda al usuario a desenvolverse mejor en el sitio y si sus elementos se muestran de una forma equilibrada o no.

- Feedback, 2 preguntas enmarcadas en la información de contactos. El usuario reconoce zonas donde encuentre formas de comunicarse sea offline $u$ online pero que este visible en el sitio.

- Utilidad, son 2 preguntas que finalizan la encuesta y lo que hace es resumir la experiencia de navegar en el sitio.

b) Características generales del tamaño del mercado: Como es un mercado de consumo masivo el tamaño del mercado es cambiante, por lo que se decide tener una aproximación del tamaño máximo del mismo. Según Kotler (2012) menciona que la demanda está relacionada directamente con el deseo de un bien o servicio y este a su vez con la capacidad de pago, el mismo autor menciona que, si el deseo como tal es innato, o por medio de herramientas de mercadeo se influyen en los mismos. Desde esa perspectiva se determina influir en la decisión de compra de productos agrícolas, para lo cual se toma un volumen mínimo y máximo de producción presentados en forma de canastas comunitarias de una de las organizaciones adheridas al MGAP, 
CIENCIAMATRIA

Revista Interdisciplinaria de Humanidades, Educación, Ciencia y Tecnología

Año VI. Vol. VI. N¹0. Enero - Julio 2020

Hecho el depósito de ley: pp201602FA4721

ISSN-L: 2542-3029; ISSN: 2610-802X

Universidad Nacional Experimental Francisco de Miranda (UNEFM). Santa Ana de Coro. Venezuela

Alfredo Mauricio Astudillo Mamarandi; Mayra Gissela Lucio Solano; Santiago Barriga

"CORPOCOSACH". las proyecciones de ventas brutas y ganancias, fueron realizados conjuntamente con la Unidad de Comercialización del Departamento de Producción Agraria de Chimborazo - MAGAP, el cual apoyó, asesoró y facilitó datos primarios.

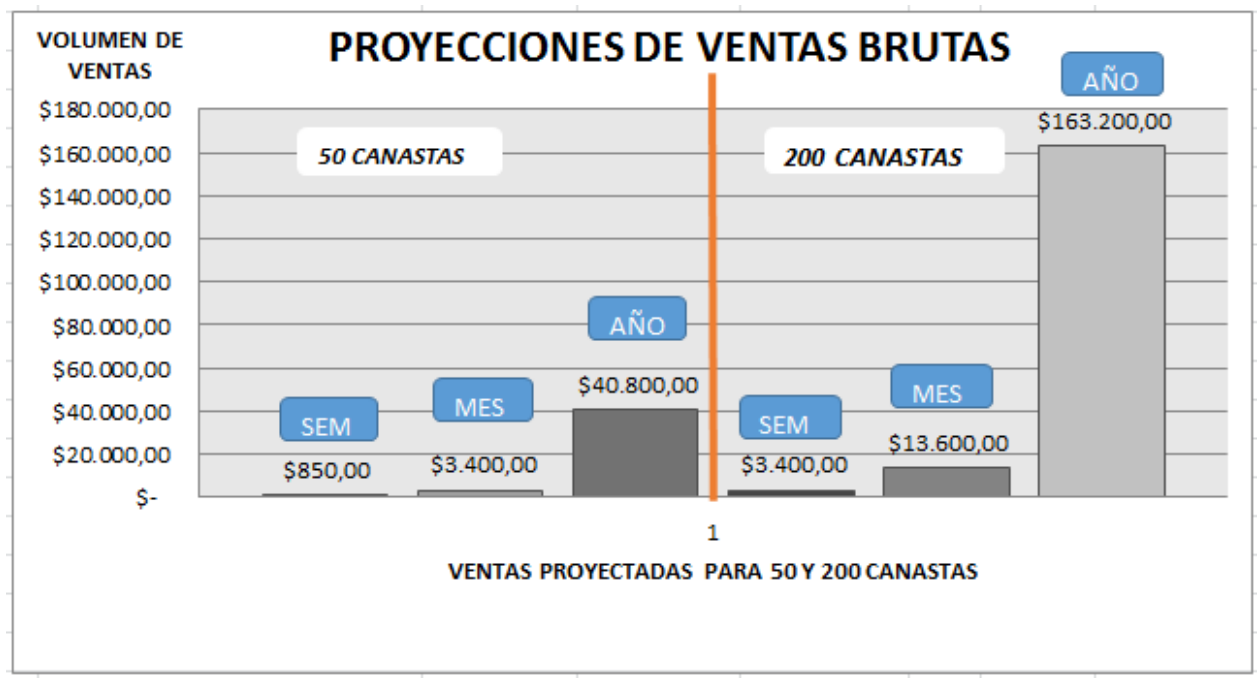

Fuente: Unidad de Comercialización del Departamento de Producción Agraria de Chimborazo - MAGAP Figura 2. Proyección de ventas brutas

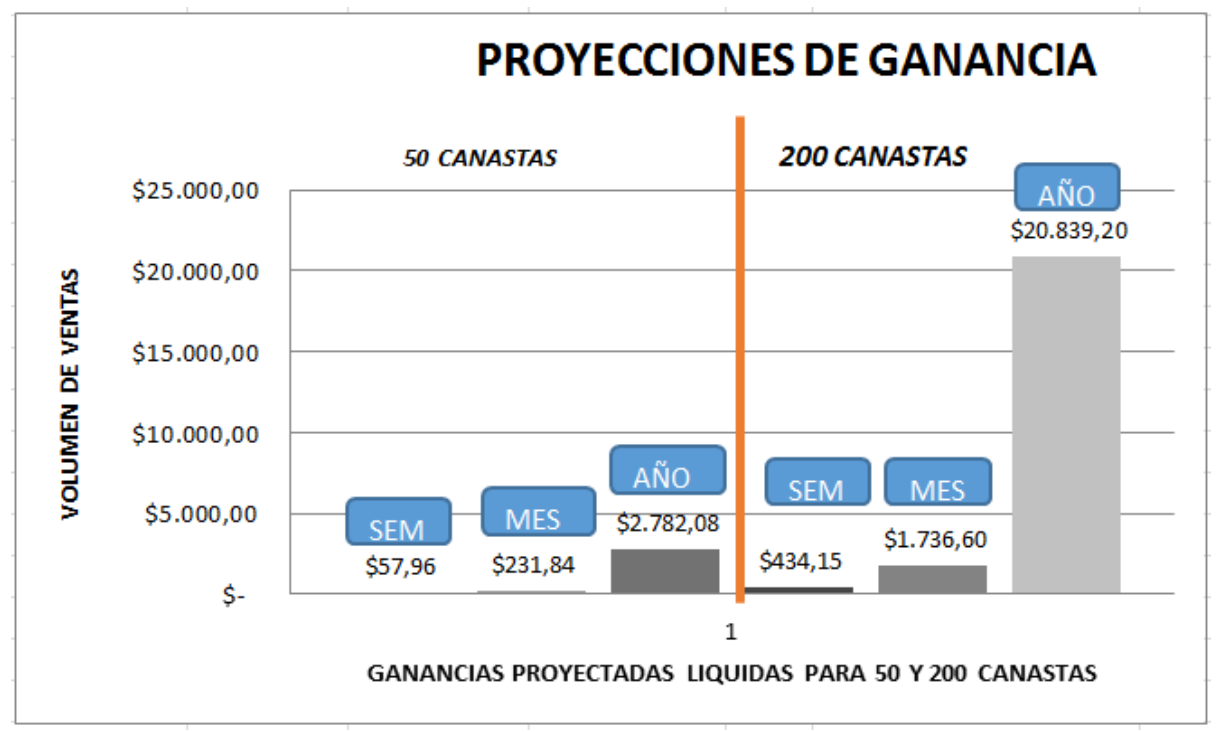


Fuente: Unidad de Comercialización del Departamento de Producción Agraria de Chimborazo - MAGAP Figura 3. Proyección de ganancia

Estos resultados fueron realizados por medio de una matriz de canastas de 24 productos con un costo aproximado de 17 USD, precio al público, con una variación de 4 presentaciones de canastas, costos de materia prima (valor monetario vigente en la realización del cálculo) y la proyección de ventas.

\section{Esquema metodológico e instrumental del tamaño del mercado}

Para determinar la demanda se recurre a una técnica denominada Método de Meta Objetivo propuesta por Gosende (2014). Un método cuantitativo, en el cual se plantea un objetivo de ventas que se encuentre dentro de la lógica del nivel de producción y debe estar por debajo del tamaño real del mercado. Varios profesionales en el campo del marketing online, manifiestan que "en estados Unidos, se suelen manejar porcentajes que se sitúan en ratios de conversión promedio del comercio electrónico entre 3 y el 6 por 100" y "en España, los porcentajes promedios son más bajos del 1 por 100" (Maciá, 2015).

Se debe considerar que la plataforma no está en funcionamiento por tal motivo se asumirá la segunda opción; una ratio de conversión del 1 por 100; es decir que hipotéticamente por 100 visitas a la tienda virtual se concretan una compra, por tal razón su fórmula quedaría de la siguiente manera:

Ratio de Conversión= Numero de ventas / número total de visitas por 100

c) Características generales para analizar la competencia: Hay que tener en cuenta aspectos como, los puntos fuertes y débiles de la competencia, identificar los puntos diferenciadores así también conocer el número de empresas, organismos u organizaciones que estén en el mercado al que se quiere acceder. Por los factores antes 


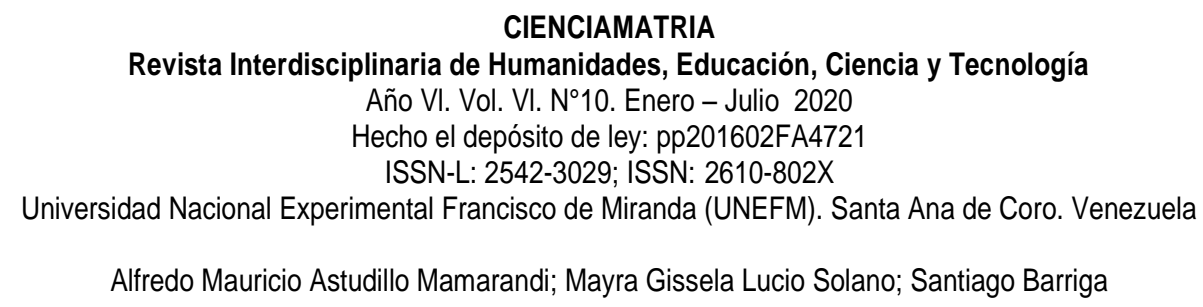

mencionados, es un estudio observacional en donde por medio de herramientas de síntesis de información se puede categorizar ciertas variables dentro del entorno de la competencia.

En el ámbito local se detectó a 13 mercados de audiencia masiva, pero solo 6 de ellos captan más del $80 \%$ de la población de la ciudad de Riobamba, según datos catastrales del GAD Municipal, se detectaron también 5 supermercados, pero por cuestiones del objetivo de estudio no se los considera ya que ellos venden productos procesados en su gran mayoría.

\section{Esquema metodológico e instrumental para el análisis de la competencia}

En este apartado se utilizó un método cualitativo y se apoyó en herramientas como, la Matriz Canvas, a modo de estrategia comparativa para determinar aspectos como: puntos débiles y fuertes de la competencia indirecta (mercados locales) además de los factores diferenciadores. $\mathrm{Y}$ las $5 \mathrm{P}$ de Porter ayudaron a analizar el mercado local y los posibles competidores directos (supermercados).

\section{RESULTADOS Y DISCUSIÓN}

Dando respuesta a los tres momentos mencionados en la metodología se presenta los resultados de la evaluación comercial, bajo el análisis de un prototipo funcional de tienda virtual:

a) Análisis de los resultados de la investigación de mercado: Con el fin de obtener datos primarios acerca de la aceptación de la ciudadanía frente a un nuevo servicio y con el objetivo de mejorar las líneas de comercialización de las organizaciones que trabajan con el MAGAP Chimborazo. Se presenta los siguientes resultados en 2 escenarios, el primero se muestra la información general e información específica y en el segundo escenario los resultados del test de usuario. 


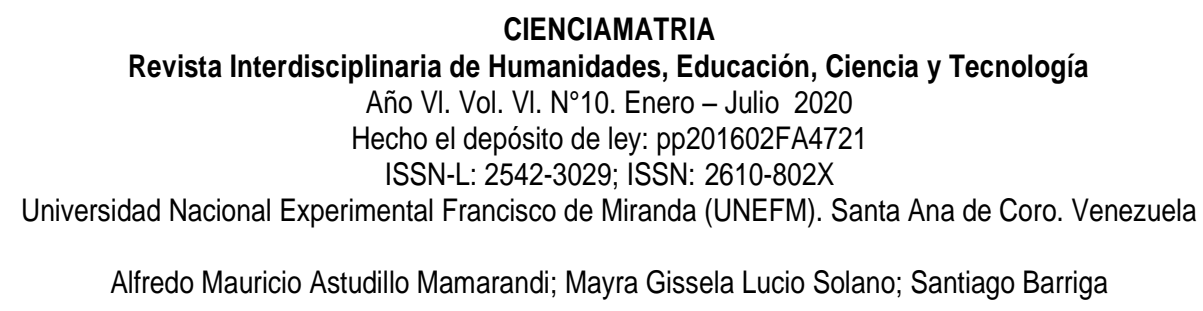

\section{Análisis de la información general:}

Por género existió una participación mayor de las mujeres con un aproximado del $73 \%$ de participación. Dentro de los encuestados aproximadamente el 50\% tiene una formación universitaria y un 30\% tiene título de posgrado y más del $72 \%$ son empleados públicos. Al mismo tiempo cumplen con los requisitos previos como es, tener experiencia en compras por internet y estar dentro de la Población Económicamente Activa.

\section{Análisis de la información específica:}

Ítem 1. ¿Las compras por internet permiten seleccionará de mejor manera productos y servicios? Análisis: Según los datos obtenidos, la mayoría de los encuestados (57\%) está de acuerdo que una compra por internet ayuda a seleccionar de mejor manera un producto o servicio y un $21 \%$ no lo considera así y/o se muestra indiferente.

Ítem 2. ¿Una tienda Virtual puede ser utilizada como canal de comercialización de productor de primera necesidad? Análisis: Como se mencionó anteriormente en el entorno donde se quiere desarrollar este plan de negocio, no es muy común la compra de productos cotidianos por medios digitales. Sin embargo, más del $50 \%$ de las personas encuestadas manifiestan estar de acuerdo con la comercialización de productos de primera necesidad y un $24 \%$ no está de acuerdo o se siente indiferente a este servicio.

Ítem 3. ¿Considera usted que la creación de una tienda virtual de productos agrícolas ayudaría a dinamizar el comercio es nuestra ciudad? Análisis: si se une los resultados de las personas que están de acuerdo y totalmente de acuerdo, tenemos un $81 \%$ de aceptación, eso hace entender que hay personas que pueden y quieren acceder a un servicio virtual donde se ofrezca productos agrícolas. Esto se da por muchos factores, como tiempo, calidad, garantía y costos. Además, algo que destacar, en su mayoría son empleados públicos y privados, su tiempo es limitado para hacer ese tipo de compra y porque solo designan un día a la semana (sábado por lo general) para realizar compras en los lugares identificados como plazas populares o supermercados de la ciudad. 


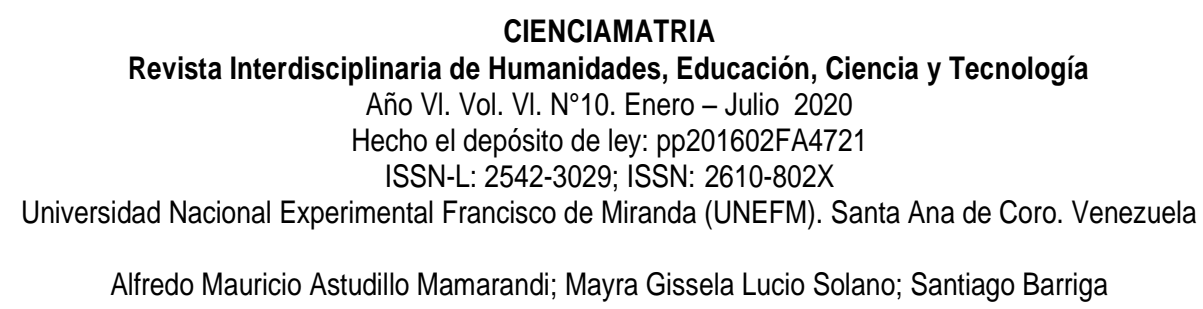

Ítem 4. ¿Si la tienda virtual de productos agrícolas, lo auspicia una institución pública como el MAGAP lo utilizaría como medio de compra habitual? Análisis: Esto hace pensar que, si existe un nicho de mercado en la ciudad, o que la presencia de un estamento gubernamental hace que la gente tenga confianza en acceder a un servicio en línea. Al igual que la pregunta anterior se une los que están de acuerdo y totalmente de acuerdo ellos juntos llegan a un $71 \%$, frente a un $29 \%$ que no está de acuerdo o se muestra indiferente.

Ítem 5. ¿Usted cree que, por comprar por la tienda virtual auspiciada por el MAGAP, los productos agrícolas llegarán de mejor calidad y certificados? Análisis: Un 40\% está de acuerdo y un $26 \%$ está totalmente de acuerdo, al pensar que los productos ofertados por la tienda virtual y auspiciada por el MAGAP son o deben ser de calidad y certificados. Las personas lo asocian a la calidad porque conocen de la actividad del MAGAP en cuanto a promover las ferias denominadas "Yo Prefiero". Un determinador común en las personas que respondieron favorablemente a la pregunta, sabías que el MAGAP realiza periódicamente las ferias antes mencionadas, pero que para ellos era muy difícil asistir por cuestiones de horario y de trabajo.

Ítem 6. ¿Podría afirmar que la presentación y la descripción detallada del producto agrícolas mostrados en la tienda virtual, ayudarían a su decisión de compra? Análisis: El aspecto de los productos y el detalle del mismo (de acuerdo + totalmente de acuerdo = $79 \%$ ) tienen mucho que ver en la decisión de compra de los usuarios. Esto se debe tomar muy en cuenta al momento de presentar la tienda virtual, pero también debe estar corroborado con el producto final que se entrega al usuario. Es decir que lo que se ve en la vitrina virtual debe tener el mayor parecido en la vida real. Así no se ocasionará clientes fugaces y pérdida de competitividad.

Ítem 7. Para ayudar a reducir tiempo de búsqueda por la tienda virtual, ¿Considera prudente la presentación de canastas elaboradas con productos agrícolas? Análisis: Va tomando más fuerza la presentación de canastas elaboradas, aunque es un tema muy 


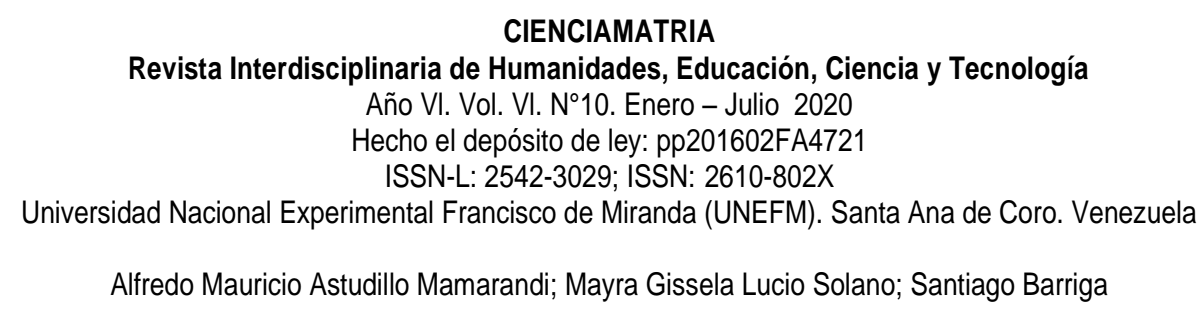

complejo, pero denota importancia al momento de elegir una compra por medio virtual. Más del $50 \%$ de los encuestados está totalmente de acuerdo en este tipo de presentación y esto va a estar determinado por gustos, preferencias y sobre todo en la capacidad de producción.

Ítem 8. Considerando que el servicio puerta a puerta es un valor agregado en cualquier comercio. ¿Cree usted que debe ser recompensado con un costo adicional? Análisis: Más del $80 \%$ rechaza el cobro adicional de la entrega de productos denominado de puerta a puerta. Este factor se debe considerar puertas adentro, es decir si se decide llegar a respetar estos datos, el costo de envió se los recargará a los productos, tanto en presentación individual o en presentación de canastas solidarias de forma que no incremente demasiado el precio; o caso contrario se debe clarificar quien se hace cargo de esos rubros, ya que este adicional al servicio puede ser un factor diferenciador con lo que respecta a la competencia así que no se le debe tomar a la ligera el resultado de la pregunta.

Ítem 9. ¿Consideraría el tiempo de entrega de los productos agrícolas, como máximo de 48 horas si lo hace vía tienda virtual? Análisis: El $75 \%$ de los encuestados no acepta el tiemplo estimado de entrega propuesto por sugerencia de los productores asociados al MAGAP. Esto indica que se debe hacer una revisión a los canales de comercialización por parte de las organizaciones productoras, porque si no pueden reducir el tiempo de entrega las ventas no efectivizan y esto puede ocasionar un declive prematuro dentro del ciclo de vida del servicio. En todo caso es una decisión que se debe analizar puertas adentro de las organizaciones que intervienen en el proyecto.

Ítem 10. Generalmente los mercados locales son puntos habituales de compra de productos agrícolas, ¿considera que ellos dan un mejor servicio en presentación y cantidad de los productos que expenden? Análisis: casi el $90 \%$ de los encuestados no está satisfecho en la presentación y servicio que brindan los mercados populares en la ciudad de Riobamba. Se pudo detectar en la mayoría de las personas se molesta al salir 


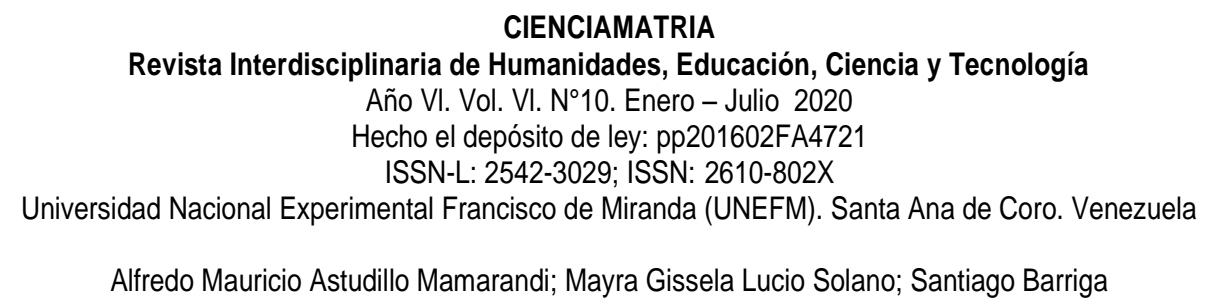

de compras, sobre todo en días de feria y no es porque el producto sea malo o llegue en malas condiciones, es porque no hay un precio y peso referencial. El comprador común debe saber detectar o tener intuición en el momento de la compra en cuanto al peso y cantidad y a eso se le añade la aglomeración de gente.

Ítem 11. ¿Considera usted que como consumidor merece precios justos y sin intermediarios? Análisis: La pregunta fue estratégica, porque puede servir para plantear un concepto de campaña publicitaria en una etapa de introducción del producto, se sabe que los intermediarios acrecientan los precios, se sabe que los productores no se llevan un porcentaje significativo de la venta de los productos y esto se corrobora ya que $90 \%$ está de acuerdo con que se debe implementar políticas de precio justo y menos intermediación.

\section{Análisis de Resultados de Reactivos del Test de Usabilidad (segundo escenario)}

A continuación, se muestran los resultados del test de usabilidad. Y tomando en cuenta el volumen de la muestra, el prototipo funcional de la tienda virtual se presentó en imagen de alta resolución (mock-up). En mucho de los casos se accedió por medio de dispositivos móviles como Tablet y celulares accediendo al link "https://alfredoma23.wixsite.com/yoprefiero".

Reactivos de identidad, Análisis: El sitio web en su parte inicial se identifica por medio de elementos en relación al URL, el logo y cromática de forma positiva, además se lo asemejan a una audiencia joven adulta con una aceptación del $44 \%$ de los encuestados. Puede estar dado por diferentes factores como: la presentación y distribución de cada elemento. Es así que más del $70 \%$ distingue a que institución pertenece el sitio, el $66 \%$ aproximadamente se le hace fácil recordar la URL, pero relacionarla a una tienda virtual se menciona que no, sin embargo, la marca "Yo Prefiero" como logo central y por su gran acogida, por las actividades que se hacen con la misma, crea una relación directa con la 


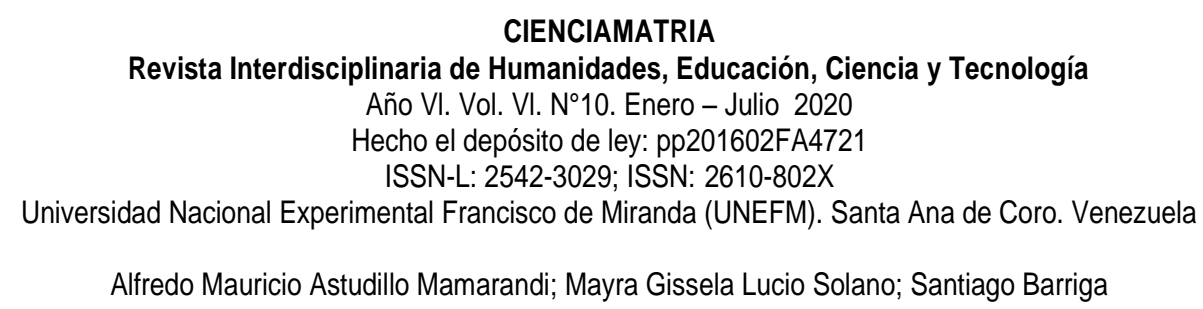

actividad del sitio, esto da a pensar que en primera instancia no existiría cambios estructurales en las maquetas presentadas.

Reactivos de contenido, Análisis: las personas encuestadas, en más del 70\%, tuvieron dificultades con respecto a la información detallada de los productos, ya que su despliegue era en forma de cuadriculas y las imágenes ocupaban demasiado espacio entre sí. En cuanto a los enlaces, mencionan que son descriptivos y funcionales por su ubicación y la implementación de la cromática llegando a tener una aceptación aproximada de $75 \%$. Al seleccionar X producto y observar su presentación, a la audiencia le parece que si es apropiada y de fácil consulta obteniendo un 65.7\%. La tipografía es procedente, legible para su lectura rápida y está dado no solo por el tipo de letra, sino por la abreviación que se utiliza en cada contexto. Y, por último, la presentación de infoproductos y su información relevante, como el título del producto, peso, tipos de volúmenes, disposición de stock, entre otras, en forma individual y en presentación de canastas alcanzó una aceptación de casi el 90\% de los encuestados.

Reactivos de navegación, Análisis: la navegación intuitiva es muy importante en cualquier proyecto web, en este caso al aplicar una cromática, tipografía y disponibilidad del elemento en el espacio web se hace adecuada, llegando a tener una aceptación en los controles de navegabilidad en un $68 \%$ de los encuestados. Esto puede estar dado por factores como incidencia en la compra frecuente en línea, el medio que utiliza para realizar compras, etc. Por otro lado, la navegación se complica cuando se sigue abriendo más pantallas, el 78\% aproximadamente tuvo dificultades dentro del sitio para reconocer en donde estaba y como regresar a un sitio específico; en el caso de los botones de acceso a otras páginas web, dentro del mismo sitio son identificados en la mayoría de las personas. Es evidente que los íconos utilizados para enlazar con las redes sociales son universales y de fácil ubicación, ya que dentro de un entorno web pueden presentarse en 


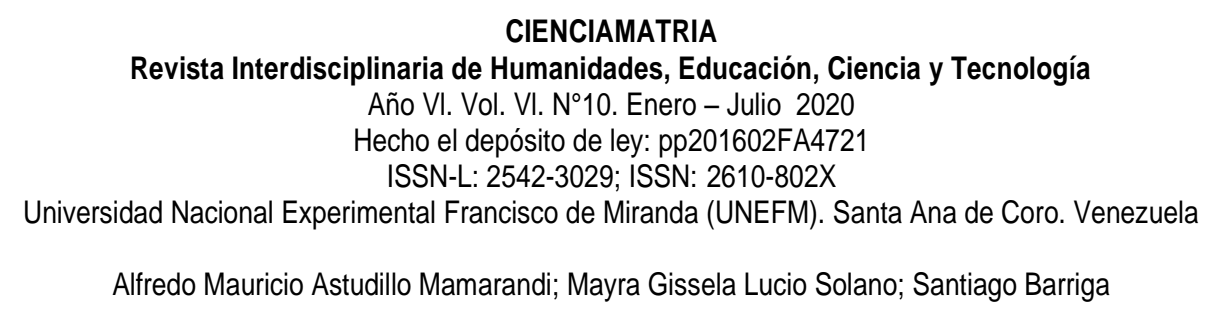

forma de iconos, texto o botón. En el caso explícito del proyecto se lo presento como un icono lateral flotante en el caso de dispositivos móviles. El carro de compras se presenta en forma de ícono así se hace fácil e intuitivo su uso, eso lo corrobora la encuesta con casi un $90 \%$ de aceptación. Y por último y no menos importante dentro de este ámbito se toma en cuenta a la lupa, la cual es universalmente reconocida para realizar búsquedas en el entorno web, y así lo relacionó las personas con un $88.6 \%$.

Reactivos de Grafica WEB, Análisis: en este ámbito de estudio no se quiso profundizar desde la parte técnica, sino más bien desde la parte estética, el cómo lo ve el usuario común. En consecuencia, se aprueba los colores, imágenes y su relación con la distribución del espacio, siente que está todo es su lugar. Con un $99 \%$ aproximadamente se llega a determinar que el diseño es atractivo y que las imágenes llaman a la acción por estar relacionadas con lo que quiere decir o llegar al usuario, hay relación imagen texto. $\mathrm{Y}$ esto desemboca en la visión de los encuestados, al decir que considera equilibrado el sitio web llegando a tener una aceptación del $85 \%$.

Reactivos de Feedback, Análisis: es claro que la aplicación del footer en toda página web es esencial, ya que es donde se puede encontrar la retroalimentación en cuanto a contactos externos, así como enlaces a redes sociales. Así lo demuestran los usuarios al reconocer dicha información, con más del $90 \%$. Esto es positivo porque ayuda a crear líneas de comunicación directa. En el sentido de control sobre el interfaz, de forma visual por parte del usuario se menciona que es efectivo, superando más del $65 \%$ de los encuestados. Pueden estar relacionado a la familiaridad de una tienda virtual común, en donde se ofertan productos de cualquier índole, eso hace reconocer de forma directa el entorno de la página web. 


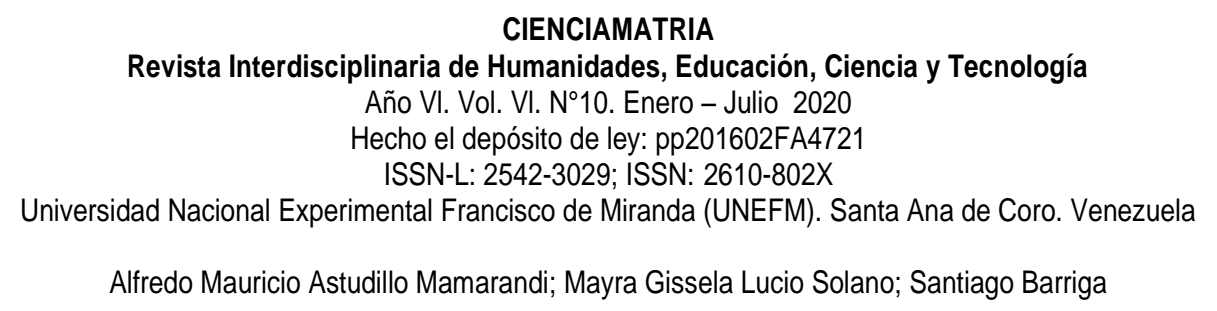

Reactivos de Utilidad, Análisis: En cuanto a la percepción del usuario y el objetivo del sitio, hay un sentido dividido. El 43.9\% manifiestas que el objetivo de la página es de uso social de servicio, mientras que más del $50 \%$ manifiesta que es de comercio. Está claro que los dos puntos observados se asemejan en la actividad, pero se debe definir como social de servicio. En la actualidad es lo que hace el MAGAP como intermediario para la venta de productos agrícolas de las asociaciones afiliadas a dicha institución. En lo que tiene que ver con el contenido y servicio que muestra el sitio a los usuarios, lo encuentras muy útil por ser innovador, concluyente y de participación entre actores sociales y productivos.

b) Análisis de los resultados del estudio del tamaño del mercado: La ciudad de Riobamba, es considerada mediana, basado en la distribución poblacional en el territorio según Censo de Población y Vivienda 2010 (Ministerio de Desarrollo Urbano y Vivienda, 2015). Sus factores se determinan por el poco desarrollo económico en el ámbito industrial y tecnológico, y su fuerza laboral está más involucrada en el ámbito público que privado. Sin embargo, existe un movimiento económico moderado; dentro del cual está el turismo, el comercio, 2 instituciones superiores de tercer nivel, la producción de artesanías y la producción agrícola por las características de su suelo y de su fuerza campesina. En base a lo dicho en la fase metodológica se procede a valorar el meta objetivo dentro del mercado local, partiendo del volumen mínimo y máximo de producción de una organización de productores agrícolas asociado al MAGAP:

Producción mínima: el objetivo es vender canastas con 24 productos agrícolas, en 4 presentaciones (detallados en el anexo 8). El objetivo de venta de la tienda virtual es vender 40.800 dólares al año.

- $\quad$ El precio promedio por canasta es de 17 dólares, para poder obtener 40.800 dólares al año se deben vender unas 2.400 canastas en el mismo tiempo. 
- Tomando en cuenta el factor hipotético de conversión (1 compra por 100 visitas) y aplicando la fórmula, sería necesario que la tienda Virtual obtenga 240.000 visitas al año, (20.000 al mes y 5.000 a la semana)

Entonces el objetivo de venta, para la producción Mínima será de 240.00 visitas al año, a la tienda virtual y eso se convertirá en 2.400 ventas anuales.

Producción máxima: El objetivo es vender canastas con 24 productos agrícolas en 4 presentaciones (detallados en el anexo 8). El objetivo de venta de la tienda virtual es vender 163.200 dólares.

- $\quad$ El precio promedio por canasta es de 17 dólares, para poder obtener 163.200 dólares al año se deben vender unas 9.600 canastas en el mismo tiempo.

- Tomando en cuenta el factor hipotético de conversión (1 compra por 100 visitas) y aplicando la formula, sería necesario que la tienda Virtual obtenga 960.000 visitas al año, (80.000 al mes y 20.000 a la semana)

Entonces el objetivo de venta, para la producción Máxima será de 960.000 visitas al año, a la tienda virtual y eso se convertirá en 9.600 ventas anuales.

Para poder cubrir los objetivos de venta en los 2 aspectos antes mencionados, se depende directamente de las actividades de promoción que se haga en un futuro. Como se puede identificar, la propuesta de la tienda virtual, está en una fase de introducción con respecto al ciclo de vida del producto, así que se debe sumar muchos esfuerzos para ingresar al mercado y conseguir dichos objetivos de venta. Cabe indicar que el calcular la producción, volúmenes de venta y periodos de cosecha de productos que son estacionales, los analiza constantemente la unidad de comercialización del MAGAP, Ios técnicos de cada organización y los productores asociados a la misma.

c) Resultados del análisis de la competencia.: Al aplicar un análisis comparativo (Figura 3), se determinó que los mercados tradicionales no cambian su forma de venta al 
público, ya que al asistir a la mayoría de estos mercados se pudo palpar que no existe información clara acerca de: precios referenciales, peso y presentación de productos. El usuario sigue acudiendo a los lugares de acopio (mercados), para adquirir sus productos agrícolas, sabiendo que la mayoría de vendedores son intermediarios. Los horarios de atención son irregulares, esto se da por denominados "días de feria", provocando un caos en cuanto a la movilidad de vehículos y peatones, sus estructuras físicas son muy estrechas e ineficientes en momentos de alto tráfico.

Para un mayor entendimiento se plantea que los literales "a" pertenecen a los mercados tradicionales y los literales "b" a la propuesta de la tienda virtual. A continuación, presentamos una síntesis del análisis que se realizó por medio de la matriz canvas, propuesta por Osterwalder's

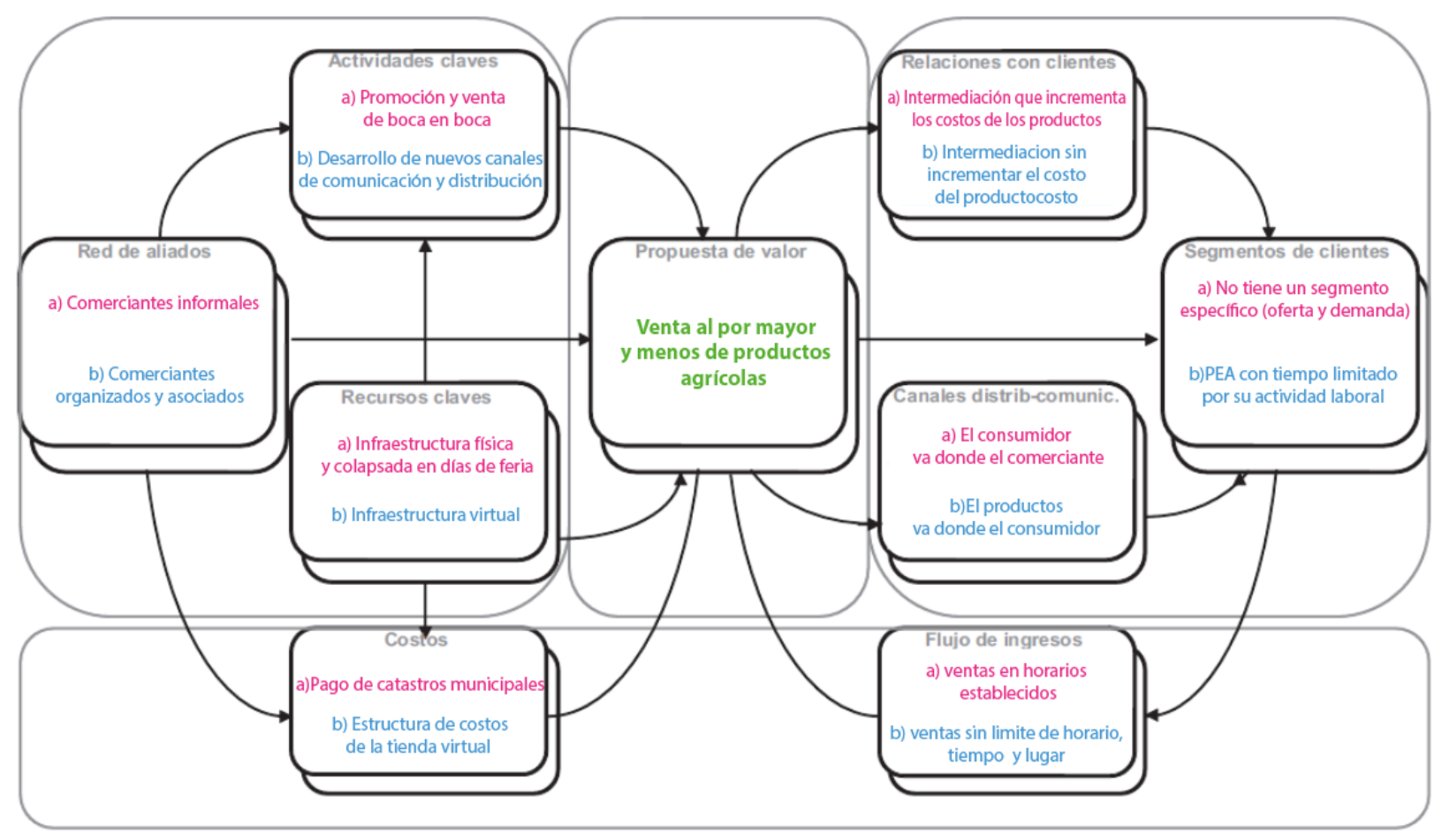

Figura 3. CANVAS como herramienta para el análisis comparativo 


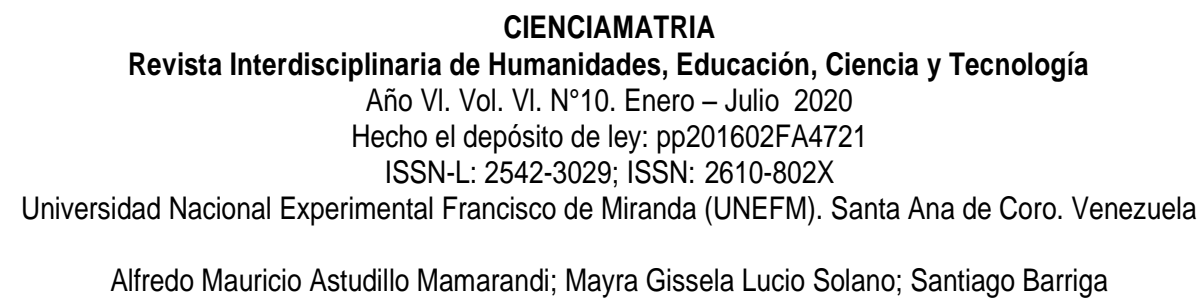

A continuación, se presenta el análisis, determinado por el investigador en base a la aplicación de las 5 fuerzas de Porter aplicado al MAGAP y su pretensión de implementar un nuevo canal de comercialización usando la tecnología.

EI Poder de negociación de los proveedores: EI MAGAP como ente intermediario sin fines de lucro, dependerá exclusivamente de los niveles de producción de las organizaciones agrícolas adheridas a la institución antes mencionada, y viceversa, los productores dependerán de la capacidad que tenga la plataforma virtual para poder presentar los productos al consumidor final.

El poder de comercialización de los compradores: Los compradores tienen poder de negociación por estar considerados dentro de la Población Económicamente Activa, distribuidos en instituciones públicas y privadas, personas con poder adquisitivo medio alto y con poca disponibilidad de tiempo para efectuar compras cotidianas, además el MAGAP tiene alto poder de negociación, por sus campañas de venta de productos agrícolas dentro de su proyecto de redes comerciales, como son las ferias populares en lugares estratégicos.

Amenaza de nuevos entrantes, o barreras de entrada: Como no existen barreras de entrada rigurosas en el comercio electrónico dentro del Ecuador, es probable que siempre esté amenazada por la implementación de este servicio por parte de una empresa privada con proveedores de mayor capacidad de producción. Es primordial que el MAGAP mantenga la reputación dentro de la ciudadanía, que sus esfuerzos de ventas y publicidad estén ligados al desarrollo de los pequeños y medianos productores. También, que las redes de productores agrícolas cumplan a plena cabalidad en la presentación, entrega y calidad de cada producto y que se estime los tiempos de entrega en base a la necesidad 
del cliente y a la capacidad de los productores para hacer llegar la mercadería en buen estado al consumidor final.

Amenaza de productos sustitutos: En gran medida los productos sustitutos están disponible en los mercados locales, pero al ser de consumo masivo, es prudente que la institución intermediaria diversifique la presentación de los productos, mejore la accesibilidad y adaptabilidad de la plataforma virtual y genere alto tráfico de visita. Y que los productores incrementen la gama de productos, haciendo que otros productores se adhieran para fortalecer la línea de comercialización virtual.

Rivalidad entre los competidores existentes: El mercado es muy amplio y la cuota que se pretende obtener de la misma está marcado por el volumen de producción y factores como la oferta y la demanda, pero el factor más diferenciador es el precio de los productos. Como se sabe mientras más intermediarios existe, más se incrementa los precios y en el caso del MAGAP una de sus intenciones es precios bajos y reducción al mínimo de intermediarios. Es un factor decisivo para marcar territorio en un mercado virtual y con productos de consumo masivo.

\section{CONCLUSIÓN}

En los apartados anteriores se identificó el tipo de servicio para la nueva línea de comercialización, definió el modelo y el tipo de negocio, en base a experiencias de comercialización y así se logró definir un esquema de funcionamiento del sitio. La muestra encuestada tiene una actitud positiva con respecto a tienda virtual auspiciada por el MAGAP; el test de usabilidad no sugiere cambios sustanciales de fondo sino de forma demostrando que el sitio es amigable con el usuario. Además, el tamaño del mercado está en función al volumen de producción y en aspectos competitivos, no existe 
competencia directa o similar en venta de productos agrícolas por medio de la plataforma digital en la ciudad de Riobamba.

Esto hace pensar en una factibilidad clara dentro del ámbito comercial que puede estar dado por su naturaleza en un mercado creciente y poco innovado en el tiempo. Se tiene claro que el objetivo primordial del modelo de negocios es conseguir ventas a través de la plataforma virtual para disminuir la intermediación, pero antes, se debe determinar claramente el cliente potencial y el mercado adecuado y por lo mencionado en el proceso de la investigación se debe identificar lo siguiente:

- Dentro del género podemos mencionar que el modelo de negocio planteado está dado para hombres y mujeres, por ser un tipo de comercialización de productos de consumo masivo no hay distinción de género, cultura o religión.

- En cuanto al rango de edad, se sugiere que sea mayor de edad y que tenga independencia económica, en el mejor de los casos y por cuestiones de manejo de diferentes formas de pago que se propone en la tienda virtual.

- En lo que tiene que ver con el nivel socio económico no se lo limita a cierto segmento del mercado, es abierto a todo público que pueda pagar por este servicio y los productos ofertados. En cierta forma a personas con un poder adquisitivo medio, medio alto.

- Dentro del perfil del usuario se puede segmentar a grupos de personas con poco tiempo disponible para realizar compras de forma convencional. Además, que tengan un dominio básico dentro del manejo del interfaz informático y que en su diario vivir estén enganchados al internet.

Otro factor a tratar en la investigación, son las estrategias posibles en el ámbito comercial, para cubrir los puntos antes mencionados. Es importante revisar algunas sugerencias que presenta un estudio denominado "Hábitos Digitales en Ecuador" presentado por la consultora internacional Interactive Advertising Bureau (IAB) en el año 2014. En el cual menciona, que el principal dispositivo de conexión a internet es la laptop, además que el 


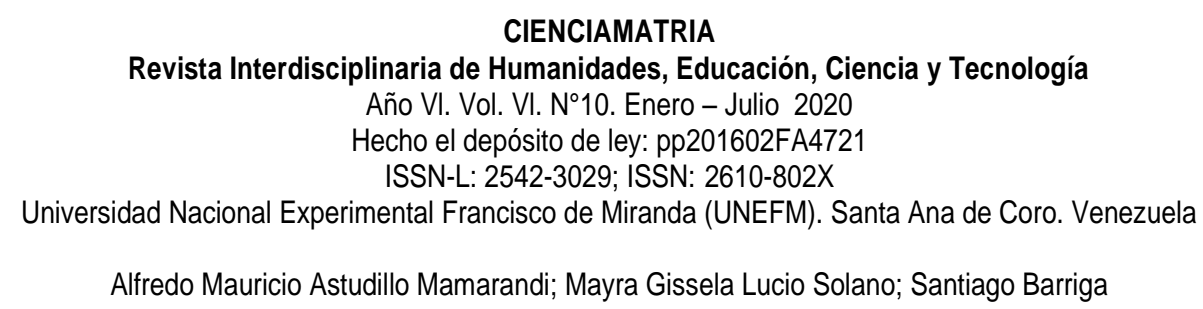

rango de edad de 19 a 32 años son los más adictos a la red, sea pos su disponibilidad de tiempo, autonomía y poder adquisitivo ya que según el estudio los coloca en un nivel socioeconómico alto y medio alto. Hay que considerar que la casa y el trabajo son los lugares donde más se conectan al internet. Los sitios más visitados son los correos electrónicos, redes sociales buscadores y que el tiempo de conexión promedio es de más de 5 horas diarias (IAB. Ecuador, 2014).

Con estos antecedentes se puede planear estrategias a llevar a cabo para posicionar la tienda virtual. Pero también se toma en cuenta la situación del MAGAP como ente auspiciador. Sus recursos son limitados y de engorrosos trámites burocráticos para asignaciones presupuestarias, por tal motivo este proyecto sugiere estrategias de generación de tráfico efectivas y sin costo de inversión monetaria sino de tiempo y esfuerzos de contenido. Al tener un departamento con profesionales dentro del área de comunicación será muy útil la breve descripción gráfica para que sea aplicado en su momento.

Lo óptimo es colocar en una matriz varias propuestas y sus herramientas adecuadas, así con el pasar del tiempo arrojaran datos cuantificables y medibles para ser analizados y restructurados de ser necesario (Maciá 2015, p. 204). Tu marca es lo que tus consumidores creen que es" (Healey 2009, p. 10) por lo tanto es importante impulsar las recomendaciones de clientes satisfechos a otros clientes potenciales dentro del mercado digital comúnmente llamado "boca a boca" o campañas evangelizadoras. Se toma en cuenta este concepto, porque el usuario o el consumidor final cree menos en la publicidad tradicional (IEBS, 2015).

\section{REFERENCIAS CONSULTADAS}

1. Almazán, F., \& Camus, J. (s.f.). Modelo de Test de Usuario. Obtenido de http://www.guíadigital.gob.cl/guía-v2/capítulos/05/anexos/pauta-test-usuario.pdf 


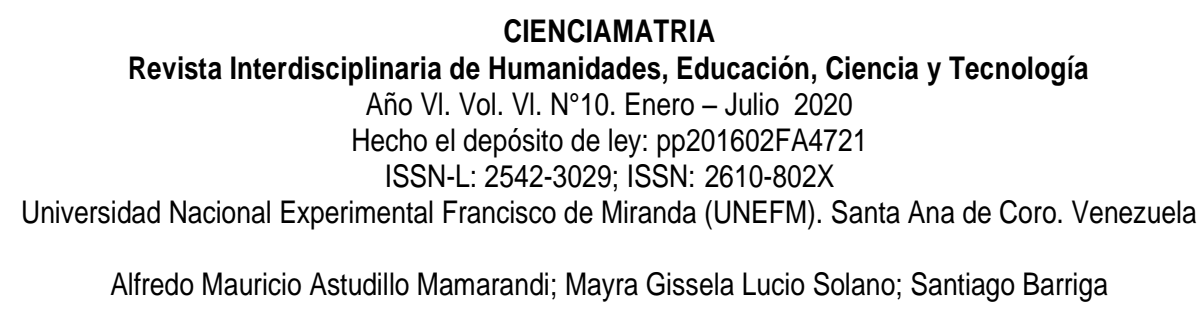

2. Cámara, R. (04 de Abril de 2017). Estadísticas de uso de internet en Ecuador y el mundo. Obtenido de INVESTICs: https://robertocamana.wordpress.com/

3. Catalán Vega, M. A. (2014). Metodologías de Evaluación de Interfaces Gráficas de Usuarios. España: eprints.rclis.org.

4. Colina, M. (2018). Ambiente virtual de aprendizaje de contabilidad ii para las horas de trabajo independiente del programa nacional de formación en administración. Revista Arbitrada Interdisciplinaria Koinonía, 2(3), 92-108. Recuperado de http://fundacionkoinonia.com.ve/ojs/index.php/revistakoinonia/article/view/54/41

5. Chakravorti, B., \& Shankar Chaturvedi, R. (28 de 09 de 2017). DIGITAL PLANET 2017. New York: The Fletcher School. Obtenido de http://artyco.com.

6. GAD Provincial de Chimborazo. (2015). PLAN DE DESARROLLO Y ORDENAMIENTO TERRITORIAL DE LA PROVINCIA DEL CHIMBORAZO 2015. Obtenido de http://www.chimborazo.gob.ec/

7. Gonzáles, M. P., Pascual, A., \& Lores, J. (2006). Evaluación Heurística. Granada: AIPO.

8. Healey, M. (2009). Que es el Branding. BArcelona: Gustavo Gili.

9. IAB. Ecuador. (2014). Estudio de Hábitos Digitales del Ecuador. Obtenido de online.fliphtml5.com: http://online.fliphtml5.com

10. IEBS. (10 de 12 de 2015). iebschool. Obtenido de https://www.iebschool.com

11. INEC. (2016). Tecnologías de la Información y Comunicaciones (TIC'S) 2016. Quito: ecuadorencifras.gob.ec. Obtenido de ecuadorencifras.gob.ec: http://www.ecuadorencifras.gob.ec

12. Jacquer, J. (2009). Marketing Estratégico. Madrid: ESIC.

13. Kotler, P., \& Keller, K. (2006). Dirección de Marketing. México: PEARSON.

14. Maciá, F. (2015). Marketing Online 2.0. Madrid: ANAYA.

15. MAGAP. (2016). La política agropecuaria ecuatoriana al 2025. Obtenido de http://www.competencias.gob.ec/wp-content/uploads/2017/05/01PPP2016POLITICA01.pdf 


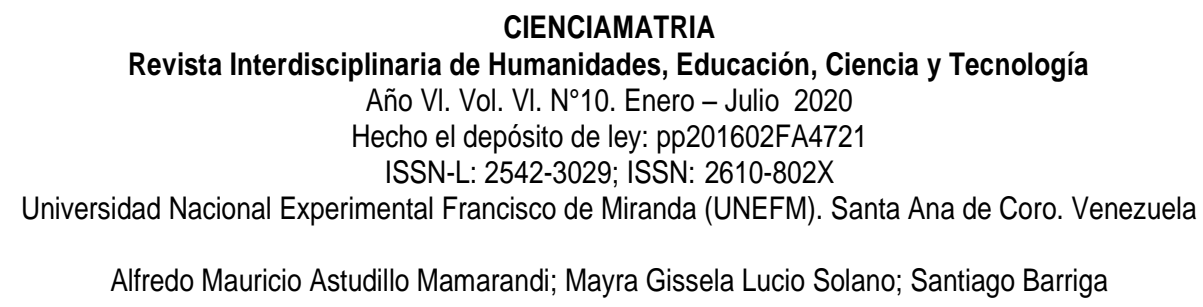

16. Mora, Q. (13 de 02 de 2016). infoautonomos.eleconomista.es. Obtenido de Viabilidad económica de tu idea de negocio online: http://infoautonomos.eleconomista.es

17. Navarro, D. (2017). Universidad de Valencia. Obtenido de http://www.uv.es/ friasnav/AlfaCronbach.pdf

18. Perozo Martín, R., \& Chirinos Martínez, A. (2019). Incidencias de la Tecnología web 2.0 en el contexto de la gobernanza y la gobernabilidad. IUSTITIA SOCIALIS, 4(6), 90-116. doi:http://dx.doi.org/10.35381/racji.v4i6.291

\section{CONSULTED REFERENCE}

1. Almazán, F., \& Camus, J. (s.f.). User Test Model. Retrieved from http: //www.guíadigital.gob.cl/guía-v2/capistros/05/anexos/pauta-test-usuario.pdf

2. Camera, R. (April 04, 2017). Internet usage statistics in Ecuador and the world. Obtained from INVESTICs: https://robertocamana.wordpress.com/

3. Catalán Vega, M. A. (2014). Evaluation Methodologies of Graphical User Interfaces. Spain: eprints.rclis.org.

4. Colina, M. (2018). Virtual accounting learning environment ii for working hours independent of the national administration training program. Interdisciplinary Arbitrated Review Koinonía, 2 (3), 92-108. Recovered from http://fundacionkoinonia.com.ve/ojs/index.php/revistakoinonia/article/view/54/41

5. Chakravorti, B., \& Shankar Chaturvedi, R. (September 28, 2017). DIGITAL PLANET 2017. New York: The Fletcher School. Obtained from http://artyco.com.

6. Provincial GAD of Chimborazo. (2015). TERRITORIAL DEVELOPMENT AND PLANNING PLAN OF THE PROVINCE OF CHIMBORAZO 2015. Obtained from http://www.chimborazo.gob.ec/

7. Gonzales, M. P., Pascual, A., \& Lorés, J. (2006). Heuristic Evaluation Granada: AIPO.

8. Healey, M. (2009). What is branding Barcelona: Gustavo Gili.

9. IAB. Ecuador. (2014). Study of Digital Habits of Ecuador. Obtained from online.fliphtml5.com: http://online.fliphtml5.com 


\author{
CIENCIAMATRIA \\ Revista Interdisciplinaria de Humanidades, Educación, Ciencia y Tecnología \\ Año Vl. Vol. VI. №10. Enero - Julio 2020 \\ Hecho el depósito de ley: pp201602FA4721 \\ ISSN-L: 2542-3029; ISSN: 2610-802X \\ Universidad Nacional Experimental Francisco de Miranda (UNEFM). Santa Ana de Coro. Venezuela \\ Alfredo Mauricio Astudillo Mamarandi; Mayra Gissela Lucio Solano; Santiago Barriga
}

10. IEBS. (10 of 12 of 2015). iebschool. Obtained from https://www.iebschool.com

11. INEC. (2016). Information Technology and Communications (TIC'S) 2016. Quito: ecuadorencifras.gob.ec. Obtained from ecuadorencifras.gob.ec: http://www.eficienterencifras.gob.ec

12. Jacquer, J. (2009). Strategic marketing. Madrid: ESIC.

13. Kotler, P., \& Keller, K. (2006). Marketing direction. Mexico: PEARSON.

14. Maciá, F. (2015). Online Marketing 2.0. Madrid: ANAYA.

15. MAGAP. (2016). Ecuadorian agricultural policy by 2025. Retrieved from http://www.competencias.gob.ec/wp-content/uploads/2017/05/01PPP2016POLITICA01.pdf

16. Mora, Q. (13 of 02 of 2016). infoautonomos.eleconomista.es. Obtained from the economic viability of your online business idea: http://infoautonomos.eleconomista.es

17. Navarro, D. (2017). University of Valencia Retrieved from http://www.uv.es/ friasnav/AlfaCronbach.pdf

18. Perozo Martín, R., \& Chirinos Martinez, A. (2019). Incidents of Web 2.0 Technology in the context of governance and governance. IUSTITIA SOCIALIS, 4 (6), 90-116. doi: http: //dx.doi.org/10.35381/racji.v4i6.291

C2019 por los autores. Este artículo es de acceso abierto y distribuido según los términos y condiciones de la licencia Creative Commons Atribución-NoComercial-Compartirlgual 4.0 Internacional (CC BY-NC-SA 4.0)

(https://creativecommons.org/licenses/by-nc-sa/4.0/). 